\title{
Missing: Outlying medical patients. If found please bleep the medics
}

\author{
Authors: Kate Edwards, ${ }^{*}$ Tom Cozens, Elen Rowlands and Rebecca John
}

\section{Introduction}

The Society for Acute Medicine has recently updated its third Clinical Quality Improvement (CQI) standard, which states that, 'All patients should be reviewed by the admitting consultant physician or an appropriate specialty consultant physician within 12 hours of arrival to hospital'. This is in line with NHS Improvement which recommends that all patients should receive a 'consultant approved care plan' within 12 hours of admission. ${ }^{2}$ This CQI is subsequently used as a benchmarking tool during the annual Society of Acute Medicine Benchmarking Audit (SAMBA). In the 2018 benchmarking audit, overall $62.8 \%$ of all patients received a consultant review within this time period. ${ }^{1}$

Within Aneurin Bevan University Health Board the statistics for time between patient admission and consultant post-take ward round (PTWR) are in line with national average. However, medical patients are still missed off PTWR due to a number of identified factors including:

> A hospital system design of a 'safari ward round' whereby medical patients are outlied to medical and surgical wards.

> A lack of 'real time' transfer data onto the clinical information technology system, Clinical Work Station (CWS).

$>$ An outdated centrally located paper patient list which is often not kept up to date, rendering patients at risk of being missed or duplicated on rounds.

Over the past 12 months there has been one mortality attributable to lack of timely consultant review after admission. This, along with many other patients who are missed, is a significant driving force for change in this respect.

\section{Materials and methods}

With Kotter's 8-step change model in mind, ${ }^{3}$ firstly an urgent climate of change was created. After discussion at a local Medical Directorate meeting, a coalition was formed including the chief registrar, associate college physicians, and college tutor/acute consultant physician.

Baseline data was collected over a 10-day period to assess the number of missed patients from PTWR, and the exact reasons

Authors: Aneurin Bevan University Health Board ${ }^{*} \mathrm{RCP}$ chief registrar for this. Subsequently, the factors discussed in the 'introduction' as most likely causes for patients being missed on PTWR were identified, and ways that these three factors could be improved upon and what would be required to make these changes were discussed.

\section{Results and discussion}

Baseline data collected prospectively over a 10-day period showed that eight patients failed to be seen on the next occurring PTWR after their admission. The majority of these patients were missed over a weekend or during the week when transferred to an outlying ward out of hours. Reasons for being missed included patient details not being documented in the patient file by the admitting doctor, and delays in updating patient location on Clinical Work Station. Of note, one patient who was an outlier at the start of the weekend failed to receive an initial consultant review until day three of admission.

With regards to changing the way patients' details are recorded, a new electronic 'watch list' was created within Clinical Work Station in a bid to improve documentation of patients awaiting PTWR. This was implemented for 24 hours to see how the process of using an electronic system worked and to identify any flaws that may need addressing. A subsequent online survey generated very positive feedback from the on-call juniors and consultants who used the system, with $100 \%$ staff stating it was very user-friendly. Feedback also highlighted how the electronic system allowed those medical on-call juniors working in emergency department to add patients without having to walk back and forth to the medical assessment unit to add them manually to the paper list.

Additionally, as a 'quick win', to aid the medical handover process from day to night teams, the postgraduate department, who were undergoing a refurbishment, gifted the handover room with two extra computers and a large TV screen to allow handover to take place electronically using the watch list.

However, the pilot study identified the ongoing issue of patients being transferred out of hours without 'real time' update on patient location. Subsequent to this, discussions are currently ongoing with the medical bed management team to aid 'real time' electronic updates of patient location once transfers have been made.

The electronic watch list is due to be enrolled into the general medical take on 1 April, once discussions are complete with the medical bed management team. Once the electronic list is implemented and bed management have updated patient 
locations electronically, a further set of data on patients being missed off PTWR will be collected prospectively and analysed to appreciate whether these changes have made a positive impact.

\section{Conclusion}

In conclusion, it is evident that patients are currently missed off post-take ward rounds due to a number of identifiable factors. There have been a number of 'quick wins' throughout the process to date, while building on these changes over time is helping drive a culture of positive change for the hospital.

\section{References}

1 Society for Acute Medicine. Society for Acute Medicine Benchmarking Audit. SAMBA 18 Report. SAM, 2019.

2 Emergency Care Intensive Support Team. Effective Approaches in Urgent and Emergency Care Paper 1. London: NHS IMAS, 2011.

3 Richie R. Using Kotter's 8-step organisational change model for success. One Clear Message, 2015. 